\title{
The expected background spectrum in NaI dark matter detectors and the DAMA result
}

\author{
V. A. Kudryavtsev 1, M. Robinson, N. J. C. Spooner \\ Department of Physics and Astronomy, University of Sheffield, Sheffield, S3 7RH, UK
}

\begin{abstract}
Detailed Monte Carlo simulations of the expected radioactive background rates and spectra in NaI crystals are presented. The obtained spectra are then compared to those measured in the DAMA/NaI and DAMA/LIBRA experiments. The simulations can be made consistent with the measured DAMA spectrum only by assuming higher than reported concentrations of some isotopes and even so leave very little room for the dark matter signal. We conclude that any interpretation of the annual modulation of the event rate observed by DAMA as a dark matter signal, should include full consideration of the background spectrum. This would significantly restrict the range of dark matter models capable of explaining the modulation effect.
\end{abstract}

Keywords: Dark matter; WIMPs; Background radiation; Radioactivity; DAMA experiment.

PACS: 95.35.+d; 14.80.Ly; 23.40.-s; 23.60.+e.

\footnotetext{
${ }^{1}$ Corresponding author; address: Department of Physics and Astronomy, University of Sheffield, Sheffield S3 7RH, UK, e-mail: v.kudryavtsev@sheffield.ac.uk
} 


\section{Introduction}

The DAMA group has reported a possible signal from dark matter particles. The first evidence came from the DAMA/NaI experiment (100 kg of $\mathrm{NaI}$ ), published in Refs. [1, 2]. The most recent papers [3, 4] based on DAMA/LIBRA measurements using $250 \mathrm{~kg}$ of $\mathrm{NaI}$ combined with the DAMA/NaI results presented improved statistical significance of the signal and evaluation of possible background sources and systematic uncertainties. The positive identification of the effect from dark matter particles is based on observation of the annual modulation of the event rate at low energies with period (one year) and phase consistent with those expected from dark matter halo models [5, 6].

Unlike many other experiments, DAMA/NaI and DAMA/LIBRA did not use pulse shape discrimination between electron and nuclear recoils to eliminate the background from gamma-rays for their high statistics exposure, relying only on the annual modulation of the total event rate and energy spectrum. In the presence of a non-zero signal, the event rate in any particular energy bin can be described by the equation:

$$
R(E, t)=b(E)+S_{0}(E)+S_{m}(E) \cos \left(\omega\left(t-t_{0}\right)\right),
$$

where $R(E, t)$ is the time-dependent event rate in a particular energy bin, $b(E)$ is the time-independent rate of background events, $S_{0}(E)$ is the time-independent nonmodulated part of the dark matter signal (average event rate), $S_{m}(E)$ is the amplitude of the modulated part of the dark matter signal, $\omega$ is the frequency of modulation ( $\omega=2 \pi / T$, where $T=1$ year) and $t_{0}$ is the time in years for the maximum of the signal. A similar equation has been used in the analysis of the DAMA data [2, 3. but the time-independent terms $b$ and $S_{0}$ of the equation were combined together. Such an approach, however, neglects the correlation between the modulated and non-modulated parts of the signal. Interpretation of the DAMA signal in terms of dark matter models carried out by the DAMA group [2, 3] and in many other papers (see, for instance [7, 8, 9] for recent analyses) were limited to fits to the modulated signal and, in some cases, to reconstruction of the average energy spectrum of the signal $S_{0}(E)$.

According to Eq. (11), the total average rate of events in each energy bin is the sum of the background rate $b(E)$ and the average rate of events from dark matter particles $S_{0}(E)$, the third term being cancelled out when averaged over a period of a year or several years. This means that if the background spectrum were known we would be able to check whether the sum of the reconstructed spectrum of the signal $\left(S_{0}(E)\right)$ and background $(b(E))$ matches the measured distribution. It turns out, however, that most efforts so far were directed to calculation of the signal (mainly $S_{m}(E)$ ) in various models helping with interpretation of the measured annual modulation effect, but totally neglecting the background contribution to the event spectrum and the information about the dark matter models that can be extracted from the measured spectrum. The background event rate and spectrum can be simulated with a reasonable degree of accuracy if the abundances of radioactive isotopes in detector materials are known. The DAMA Collaboration should have included the evaluation of this spectrum in their results to support their claim of dark matter discovery. 
Limiting the data analysis to the modulated part of the spectrum only, and ignoring information from the non-modulated component of the signal and background, severely weakens the conclusions that may be derived from the experiment. Self consistent analysis of the experimental data should include all components of the measured spectrum: background, modulated and non-modulated parts of the signal. In this way no information is missed and a proper fit of the measured spectrum to the sum of predicted distributions is possible. In practice, accurate calculation of the background spectrum in terms of the absolute event rate (events $/ \mathrm{kg} / \mathrm{day} / \mathrm{keV}$ ) can only be done with high accuracy if concentrations of radioactive isotopes in all materials in and around the detector are precisely known. However, any particular source of background radiation has a characteristic spectrum and a combination of spectra from different sources with certain weights should match observations with or without signal. Hence the difference between the measured and background spectra should be equal to the spectrum of dark matter events. Analysing the measured spectrum and simulated background would allow us to constrain the dark matter signal.

We have used our expertise in running $\mathrm{NaI}(\mathrm{Tl})$ dark matter experiment NAIAD [10] and in modelling radioactive background in different types of detectors [11, 12, 13] to simulate gamma-ray production, transport and detection in the DAMA/LIBRA experiment. In this paper we present detailed Monte Carlo simulations of the background spectrum in a modelled setup of the DAMA/LIBRA $\mathrm{NaI}(\mathrm{Tl})$ detector. We then discuss the implications that these results may have on interpretation of the signal claimed by the DAMA group. We demonstrate that the energy spectrum measured in the DAMA/LIBRA experiment can hardly include any dark matter signal by (i) subtracting some possible WIMP signals from the measured spectrum and comparing this to the simulated background and (ii) subtracting the simulated background spectrum from the measured one and showing that the difference spectrum has a minimum at low energies which would not be a usual feature for dark matter signals. We also show that some inconsistencies exist between low-energy and high-energy parts of the measured DAMA/LIBRA spectrum.

A possible problem with the background spectrum in the DAMA/NaI experiment in the presence of dark matter signal was pointed out also in Ref. [14]. Several recent analyses (see, for instance, [7, 9]) used the measured spectrum to constrain theoretical models but did not take into account the spectrum of background events that should contribute to the observed rate.

\section{Modelling of the background radiation}

To model the DAMA/LIBRA background we have considered three locations of radioactive sources: (i) external sources producing gamma-rays and other particles outside the NaI crystals; ii) internal sources of radiation evenly distributed within the volume of the crystal; and iii) surface sources with radioactive isotopes concentrated within the thin surface layer of the crystal (assumed here to be $50 \mu \mathrm{m}$ thick). 


\subsection{External background sources}

The setup of the DAMA/LIBRA experiment comprising $25 \mathrm{NaI}(\mathrm{Tl})$ crystals, $\sim 10 \mathrm{~kg}$ each, was modelled using the published geometry [3, 4]. For case (i) the source of background radiation was assumed to be in the $100 \mathrm{~g}$ envelopes of the photomultiplier tubes (PMTs) attached to the light guides connected to the two flat surfaces of the crystals. The exact position of the radiation source (PMTs, light guides or similar) is not important since only the high-energy gamma-rays can reach the crystal and the spectrum of events at low energies (below $30 \mathrm{keV}$ ) is fully determined by Compton electrons. The PMT envelopes were populated with radioactive isotopes of ${ }^{238} \mathrm{U},{ }^{232} \mathrm{Th}$ and ${ }^{40} \mathrm{~K}$. The isotopes were allowed to decay in our Monte Carlo code, based on the GEANT4 toolkit (version 9.2) [15]. All particles were generated according to GEANT4 library. All isotopes in the decay chains of ${ }^{238} \mathrm{U}$ and ${ }^{232} \mathrm{Th}$ decayed in our simulations in secular equilibrium (the spectra of gamma-rays from uranium and thorium decay chains in secular equilibrium have also been obtained in Ref. [16]). Obviously, only high-energy gamma-rays can reach $\mathrm{NaI}$ from large distances, with X-rays, electrons and alphas being absorbed in the surrounding materials.

We have also considered ${ }^{60} \mathrm{Co}$ in copper around the detectors and in a shield, as a possible source of background events. Simulations of ${ }^{60} \mathrm{Co}$ induced events have been carried out in the same way as for other isotopes.

Figure 1 shows the spectra of energy depositions from electron recoils in the crystal with PMTs contaminated with ${ }^{238} \mathrm{U},{ }^{232} \mathrm{Th}$ and ${ }^{40} \mathrm{~K}$. For normalisation we have used typical concentrations of radioactive isotopes in ultra-low background PMTs produced by ETL and used by DAMA: $30 \mathrm{ppb}$ of uranium and thorium, $60 \mathrm{ppm}$ of potassium. Events produced by ${ }^{60} \mathrm{Co}$ decays in copper are also shown in Figure 11 assuming a decay rate of ${ }^{60} \mathrm{Co}$ of $10 \mathrm{mBq} / \mathrm{kg}$. This rate is significantly higher than typically measured in low-background copper. Only events in which an energy deposition above $0.5 \mathrm{keV}$ is detected in a single crystal (close to the energy threshold of the DAMA experiment), were included. Multiple scattering events in two or more crystals with energy depositions exceeding $0.5 \mathrm{keV}$ were excluded. If a photon scattered two or more times in one crystal, all energy depositions were summed together. The energy deposition spectra were convolved with Gaussian distributions describing the energy resolution of the DAMA/LIBRA detectors [4].

As can be seen from Figure 1 the energy deposition spectra from all decay chains at low energies from external sources are essentially flat, due to Compton scattering of highenergy gamma-rays in the crystal. Spectra from any other decay outside the crystal have very similar shape at low energies dominated by Compton scattered electrons. The assumption of secular equilibrium does not affect the shape of the spectrum at low energies: the spectrum from any particular decay has the same flat shape below 30 $\mathrm{keV}$. The broad peaks at about $150 \mathrm{keV}$ are the well-known back-scatter peaks due to the scattering of photons in materials around the crystal prior to entering the sensitive volume. 


\subsection{Internal background sources}

For case (ii) the sources of background radiation were distributed evenly in all the crystals. Figure 2 shows the energy deposition spectra from ${ }^{238} \mathrm{U},{ }^{232} \mathrm{Th}$ and ${ }^{40} \mathrm{~K}$ decay chains, as well as from the decays of some other isotopes $\left({ }^{129} \mathrm{I}\right.$ and $\left.{ }^{22} \mathrm{Na}\right)$ identified as possible sources of background in the DAMA/LIBRA experiment [3, 4]. The spectra were convolved with the energy resolution function. For energy depositions from $\alpha$ particles we have assumed a quenching factor as measured by DAMA [4].

The calculated spectra were normalised using the measured concentrations of radioactive isotopes or their decay rates as reported by DAMA [4]. The main problem here is that only a range of concentrations in the different crystals can be found in Ref. [4, together with a value determined for one crystal as an example. The DAMA Collaboration reported [4] the ranges for decay rates of the isotopes ${ }^{40} \mathrm{~K},{ }^{129} \mathrm{I}$ and ${ }^{22} \mathrm{Na}$, as well as for some isotopes from the ${ }^{238} \mathrm{U}$ and ${ }^{232} \mathrm{Th}$ decay chains. We used the reported typical concentrations of these isotopes in the crystals to normalise the spectra shown in Figure 2, 5 ppt of ${ }^{238} \mathrm{U}$ (0.7 to $10 \mathrm{ppt}$ was reported in Ref. [4] assuming equilibrium), $5 \mathrm{ppt}$ of ${ }^{232} \mathrm{Th}$ (0.5 to $7.5 \mathrm{ppt}$ was reported), $10 \mathrm{ppb}$ of natural potassium ( $\leq 20 \mathrm{ppb}$ was reported), $0.2 \mathrm{ppt}$ of ${ }^{129} \mathrm{I}$ (a similar value was reported but for two crystals only) and $6.46 \times 10^{-14} \mathrm{ppb}$ of ${ }^{22} \mathrm{Na}$. The ${ }^{232} \mathrm{Th}$ decay chain was reported to be in equilibrium [4] unlike ${ }^{238} \mathrm{U}$ for which the equilibrium was found to be broken: a factor of 5.5 higher rate was found at the end of the chain indicating higher concentration of ${ }^{210} \mathrm{~Pb}$ than expected from the equilibrium. To plot the uranium spectrum in Figure 2 we assumed equilibrium in the uranium decay chain. Since the spectrum from ${ }^{238} \mathrm{U}$ chain does not have any features below $30 \mathrm{keV}$, the assumption of equilibrium should not affect the shape of the spectrum at low energies. Assuming higher concentration of ${ }^{210} \mathrm{~Pb}$ compared to the equilibrium would result in a higher peak at 40-60 keV, inconsistent with the measurements.

Present simulations were carried out using the most recent version 9.2 of GEANT4. The spectra are slightly different from those obtained with the previous version $9.1 \mathrm{p} 3$. We have checked the production of the low-energy X-rays, conversion electrons and Auger electrons in GEANT4 and have found that, despite significant improvements, some low-energy photons and electrons are missing resulting in an energy imbalance and spectrum distortions if the radioactive decays happened inside the crystal. We have corrected this by requiring the conservation of energy, with a missing energy being deposited at the point of the decay. The correction becomes significant when a low-energy beta-decay is accompanied by the emission of low-energy X-rays or electrons that are missing from the GEANT4 library, in particular for ${ }^{129} \mathrm{I}$ decay.

For case (iii) (radioactive sources distributed in the surface layer), the spectra (not shown here) are found to be similar to case (ii) with some enhancement in the number of events at low energies due to particles escaping from the crystal. This effect, however, does not significantly change the shape of spectra at low energies. The assumption that radioactive sources are located in a thin layer at the crystal surface requires their concentrations to be very high, the enhancement over the bulk concentration being equal to the ratio of bulk to surface volumes. Such high concentration looks unrealistic in a well-controlled low-background dark matter experiment. 


\section{Implications for DAMA results}

\subsection{DAMA spectrum}

In this section we will discuss how the measurements and simulations of the background radiation may help with interpretation of the effect reported by DAMA. Figure 3 shows the energy spectra reported by the DAMA/NaI (open circles) and DAMA/LIBRA (filled circles) experiments. The spectra, as reported [2, 3], were corrected for all detection and reconstruction efficiencies. The energy spectra from the DAMA/NaI and DAMA/LIBRA experiments are slightly different. This may be due to different cuts to remove PMT noise and different efficiencies in the two data sets at small energies where the rate is dominated by the PMT noise. The difference looks to be significant in the position of the peak at about $3 \mathrm{keV}$ that is due to ${ }^{40} \mathrm{~K}$ decay [4]. The energy spectra measured in other NaI detectors at low energies are different from those observed by the DAMA/NaI and DAMA/LIBRA experiments. The filled triangles show the spectrum of energy depositions as measured in NaI by the NAIAD experiment [10]. The DAMA spectra show the minimum at about $2 \mathrm{keV}$ that was not seen in NAIAD. However, the absolute rate of events was higher in NAIAD than in DAMA/LIBRA. Event rate of about 2 events $/ \mathrm{kg} /$ day $/ \mathrm{keV}$ above $5 \mathrm{keV}$ (similar to DAMA) was observed in Ref. [17] but the rate in the $3 \mathrm{keV}$ peak was higher than in DAMA.

The interaction of WIMPs with the target ( $\mathrm{Na}$ and I) material is one interpretation of the measured annual modulation effect seen by DAMA. The curve in Figure 3 shows the energy spectrum of events from spin-independent WIMP interactions with parameters $M_{W I M P}=60 \mathrm{GeV}$ and $\sigma_{S I}=7 \times 10^{-6} \mathrm{pb}$ in the isothermal halo model. These parameters are close to the best fit to the measured signal as reported in Ref. [2]. Filled squares show the difference between the measured spectrum and the spectrum of the WIMP signal described above. So the pure background spectrum should look like this curve. This 'background spectrum' shows a deep minimum at about 1.5-2.0 keV. The depth and exact position of the minimum depends on the dark matter particle and halo model but the minimum should be present in all models, most of them requiring the ratio of non-modulated to modulated parts of the signal to be at least 20-30 at 2-4 $\mathrm{keV}$. The rise of the measured event rate below $1.5 \mathrm{keV}$ is most likely due to the PMT noise that was not included in our simulations.

The rate at $4-10 \mathrm{keV}$ in the DAMA/LIBRA experiment was reported to be about 1 event $/ \mathrm{kg} /$ day $/ \mathrm{keV}$ [3, 4]. The peak at about $3 \mathrm{keV}$ is due to the intrinsic contamination of the crystals by ${ }^{40} \mathrm{~K}$ [4]. The reported rate at $20-30 \mathrm{keV}$ is about 0.5 events $/ \mathrm{kg} /$ day $/ \mathrm{keV}$. It is not clear, however, at what energy and why the event rate drops from about 1 events $/ \mathrm{kg} /$ day $/ \mathrm{keV}$ as observed at $4-10 \mathrm{keV}$ to about 0.5 events $/ \mathrm{kg} /$ day $/ \mathrm{keV}$ as observed at $20-30 \mathrm{keV}$. It is difficult to explain such a decrease in rate with our simulations. Note, however, that the DAMA Collaboration reported the spectra at 20-80 keV from two individual crystals only, whereas the spectrum below $10 \mathrm{keV}$ is averaged over all 25 crystals. If the majority of the crystals show much higher rate at 20-30 keV, then the rates below $10 \mathrm{keV}$ and above $20 \mathrm{keV}$ might be consistent. 


\subsection{Combined background spectra from simulations and com- parison with measurements}

Figure 4 (solid curve) shows the sum spectrum of simulated events in NaI crystals due to internal and external sources of background as plotted in Figures 1 (except ${ }^{60} \mathrm{Co}$ ) and 2. The aforementioned concentrations of the radioactive isotopes were used. Assuming typical concentrations of uranium, thorium and potassium in PMTs, this external source of background does not contribute substantially to the total spectrum. The simulated absolute rate below $10 \mathrm{keV}$ (solid curve in Figure 4) is significantly smaller than observed by DAMA ( 1 event $/ \mathrm{kg} / \mathrm{day} / \mathrm{keV}$, see Figure 3 above and Figure 27 in Ref. [4). If the copper of the crystal case or shielding, or other materials are contaminated by ${ }^{60} \mathrm{Co}$ with a decay rate about $500 \mathrm{mBq} / \mathrm{kg}$, then this source of radiation can explain the rate of events at $4-10 \mathrm{keV}$ as measured by DAMA. The broad peak at $40-100 \mathrm{keV}$ is due to the decay of ${ }^{129} \mathrm{I}$. The event rate in the peak is consistent with that reported by the DAMA Collaboration [4].

In an attempt to make the simulated spectrum compatible with the measurements we examined an effect of assuming higher concentration of some radioactive isotopes compared to the reported values. The flat part of the spectrum at low energies could be due to either intrinsic ${ }^{238} \mathrm{U}$ (with or without equilibrium) or any external source (decay chains of ${ }^{238} \mathrm{U}$ or ${ }^{232} \mathrm{Th},{ }^{40} \mathrm{~K},{ }^{60} \mathrm{Co}$ ). Each spectrum would result in characteristic features at higher energies.

We have first assumed that the internal contamination of the crystals with radioisotopes is higher than reported by DAMA. The dashed curve in Figure 4 shows the spectrum calculated assuming higher concentrations of certain isotopes inside the crystals compared to the values used in Figure 2: twice as much of natural potassium (20 $\mathrm{ppb})$ to bring the $3 \mathrm{keV}$ peak to the measured value, 8 times more ${ }^{238} \mathrm{U}$ (40 ppt) to make the spectrum flat at $4-10 \mathrm{keV}$ with a measured rate of about 1 event $/ \mathrm{kg} / \mathrm{day} / \mathrm{keV}$, and 4 times more ${ }^{232} \mathrm{Th}(20 \mathrm{ppt}$ ) to achieve a better match at 4-10 keV (see also Figure 5 below). An important feature of this spectrum is the presence of a peak from ${ }^{210} \mathrm{~Pb}$ decay at $\sim 40-50 \mathrm{keV}$ ( $\beta$-decay accompanied by gamma-rays and X-rays) which dominates over ${ }^{129} \mathrm{I}$ decay. If the equilibrium is broken in the uranium decay chain and the decay rate of ${ }^{210} \mathrm{~Pb}$ is higher than that of the parent isotopes as reported by DAMA [4], then the peak from ${ }^{210} \mathrm{~Pb}$ will be even more pronounced making the predicted spectrum inconsistent with the measurements (see Figure 11 of Ref. [4]).

Then we have looked at the possibility of having higher background rate due to external sources. Any external source of background radiation would produce a broad back-scatter peak centered at about $150 \mathrm{keV}$. The dotted curve in Figure 4 shows the spectrum calculated using higher contaminations of potassium in the PMTs (12000 ppm or $1.2 \%$ ) and in the crystals (20 ppb) to account for the flat part of the measured spectrum and absolute rate at low energies (including the peak at about $3 \mathrm{keV}$ ). Such a high concentration of potassium (or similarly very high concentrations of uranium or thorium) in low background PMTs looks unrealistic but is used to illustrate the effect of possible external source of background. In fact, such a source could be much closer to the crystal and not shielded by any light guides or copper. In this case the concentration needed to obtain the flat spectrum and the rate consistent with measurements 
at low energies might be smaller. For instance, as mentioned above, a high decay rate (about $0.5 \mathrm{~Bq} / \mathrm{kg}$ ) of ${ }^{60} \mathrm{Co}$ in copper around the detectors (case and shielding) could explain the observed rate at $4-10 \mathrm{keV}$.

An important feature of any spectrum dominated by external source, is the presence of a broad back-scatter peak at about $150 \mathrm{keV}$. Figure 11 in Ref. [4] shows, however, that the measured spectrum at 40-80 keV (for two individual crystals) can fully be explained by the sum of the ${ }^{129} \mathrm{I}$ and ${ }^{210} \mathrm{~Pb}$ decays leaving too little room for the back-scattered gamma-rays. A more detailed investigation of the background could be performed if the measured spectrum averaged over all crystals for a broad energy range (from $1 \mathrm{keV}$ to a few $\mathrm{MeV}$ ) were to be published by the DAMA Collaboration.

The minimum at 1.5-2 keV that should be present in the background spectrum (Figure 3) due to the contribution of the WIMP signal to the observed event rate, is not seen in the simulated spectra (Figure 4) independently of the assumptions made about radioactive contaminations in different materials in and around the detectors.

Figure 5 shows simulated spectra below $10 \mathrm{keV}$ with 'optimised' concentrations to match the measured spectrum at low energies: external background sources were assumed to be in the PMTs with concentrations specified above for Figure 1; internal sources are as for the dashed curve of Figure 2- potassium at $20 \mathrm{ppb},{ }^{129} \mathrm{I}$ at $0.2 \mathrm{ppt}$, ${ }^{238} \mathrm{U}$ at $40 \mathrm{ppt}$ and ${ }^{232} \mathrm{Th}$ at $20 \mathrm{ppt}$. The simulated spectrum is compared with the measurements without signal subtraction (open squares) and with signal subtraction. For the latter case we assumed $60 \mathrm{GeV}$ mass WIMPs with $\sigma_{S I}=7 \times 10^{-6} \mathrm{pb}$ (filled squares) and $\sigma_{S I}=2 \times 10^{-6} \mathrm{pb}$ (open circles). A similar simulated spectrum but over a wide energy range is shown in Figure 4 by the dashed curve. Even for a model with small cross-section, the measured spectrum minus signal (open circles in Figure 5 ) does not match the predictions at $1.5-2.5 \mathrm{keV}$. A better match between the simulated background spectrum and the measurements minus signal would require a very small ratio of non-modulated to modulated parts of the signal spectrum (certainly less than a factor of 10 at $2-4 \mathrm{keV})$.

Our 'optimised' background spectrum, although being in reasonable agreement with the measured one, does not satisfy a crucial requirement in the presence of a signal: the background event rate should not exceed the measured one in any energy bin since a contribution from a signal is expected too. In some energy bins in Figure 5, in particular at $2-3 \mathrm{keV}$, the calculated background rate exceeds the measured one. This can partly be corrected if we assume an uncertainty in the energy calibration of DAMA/LIBRA. For instance, shifting the experimental data by about $0.25 \mathrm{keV}$ towards low energies can improve the agreement with simulations and opens the way to seeing what the signal spectrum could look like after background being subtracted from the measured rate.

Figure 6] shows the measured DAMA/LIBRA spectrum shifted by $0.25 \mathrm{keV}$ towards low energies. (Note that this is only an assumption and other ways of improving the agreement between the measured spectrum and the background taking into account the uncertainties in energy calibration, resolution, etc are possible.) Let us now require that the expected background spectrum should be less than the measured rate in any energy bin. The red solid curve shows the background spectrum with internal concentrations of $34 \mathrm{ppt}$ of ${ }^{238} \mathrm{U}, 20 \mathrm{ppt}$ of ${ }^{232} \mathrm{Th}$ and $20 \mathrm{ppb}$ of natural potassium. 
The reduction of uranium concentration from $40 \mathrm{ppt}$ to $34 \mathrm{ppt}$ is the consequence of the above requirement. The background spectrum still looks reasonably close to the measured one and leaves practically no room for the signal, the difference between the measured spectrum and the background being shown by filled squares.

To allow for a signal to be present we should now reduce the concentrations of radioisotopes in our calculations. Reducing the internal concentration of uranium to $20 \mathrm{ppt}$ (dashed blue curve in Figure 6 corresponds to the sum spectrum) results in a 'signal' spectrum shown by open circles which is obviously inconsistent with any dark matter signal. Internal concentration of thorium does not affect the spectrum below $6 \mathrm{keV}$ at all (see Figure 5). Lowering the internal concentration of potassium to $10 \mathrm{ppb}$ reduces the background event rate at the peak only and does not affect energies above $4.5 \mathrm{keV}$. The difference between the measured and expected background spectra that can be associated with the signal, looks like a peak at about $3 \mathrm{keV}$ (filled triangles) - copying the peak from ${ }^{40} \mathrm{~K}$. If realistic dark matter models exist with such a feature and with a low ratio (about 10) of non-modulated to modulated parts of the signal spectrum, they should be carefully analysed. There may be, however, a simpler explanation of the annual modulation around $3 \mathrm{keV}$ peak related to the selection of single hit events at these energies which are dominated by ${ }^{40} \mathrm{~K}$ (see also Ref. [18] for discussion). This can be checked by looking at the time behaviour of the rate around $1460 \mathrm{keV}$ and of the coincident events with a $3 \mathrm{keV}$ energy deposition in one crystal and $1460 \mathrm{keV}$ deposition in another one. The problem with a smaller measured rate at $40-60 \mathrm{keV}$ compared to the required one from a higher concentration of ${ }^{238} \mathrm{U}$ is still present in all these scenarios.

If we do not assume high concentrations of some isotopes as suggested above, the simulated event rate at low energies is much smaller than the DAMA measurements and no dark matter signal can explain the difference above $6 \mathrm{keV}$. At present it is difficult to explain the apparent inconsistency between the low-energy part (requiring high concentrations of uranium and possibly thorium in the crystals or high rate from an external source) and the high-energy part (not showing associated features) of the DAMA spectrum.

Several fits of the measured modulated part of the signal to the predictions of different dark matter models were shown in Ref. [3]. Unfortunately, no comparison between the measured spectrum and the predictions including the background and the time-averaged signal was presented, although such a comparison would provide a very important test of the interpretation of the observed effect. For instance, most WIMP models that fit the DAMA/NaI data [2, 7, 8] (some of them are already excluded by other experiments) are not compatible with the combination of background and signal spectra as shown in the figures. Most models require the non-modulated part of the signal to be at least 20-30 times bigger than the modulated part at 2-4 keV resulting in a deep minimum after the signal spectrum is subtracted from the measured one (see Figure 5). Independently of the relative contributions of external sources of different isotopes (no accurate measurements for the DAMA experiment were found), and even varying intrinsic contaminations of the crystals, it is hard to explain such a minimum in the overall background spectrum at $1.5-2 \mathrm{keV}$, the measured rate in the peak at 3 $\mathrm{keV}$ and almost flat rate of 1 event $/ \mathrm{kg} /$ day $/ \mathrm{keV}$ at $4-10 \mathrm{keV}$ as seen in Figure 3, with our simulations. 
Accurate comparison of the simulations with the measured background spectrum in the DAMA crystals is difficult due to the absence of published data on the spectrum at all energies (from $1 \mathrm{keV}$ to a few $\mathrm{MeV}$ ). We stress, however, that any analysis of the DAMA signal should include predictions of the background spectrum and its comparison with the measured spectrum minus the averaged dark matter signal extracted from the annual modulation effect.

Our results are practically independent of the assumption about the channeling effect in $\mathrm{NaI}$ crystals (see [3] and references therein). Our conclusions assume that dark matter models predict a high ratio of the non-modulated to modulated parts of the spectrum (only a small fraction of spectrum is modulated) which does not depend much on the channeling effect.

\section{Conclusions}

We have presented Monte Carlo simulations of the background rates and spectra due to different sources expected in the DAMA/LIBRA NaI crystals. Any specific source of background radiation has a characteristic spectrum and, ideally, the measured spectrum should be consistent with a combination of predicted spectra from the different sources. We have found that it is hard to match the measured spectrum of DAMA/LIBRA at low energies (even without signal) with the simulated background assuming measured concentrations of radioactive isotopes in NaI crystals. Some features (drop in observed intensity between 10 and $20 \mathrm{keV}$, low total rate from simulations) are difficult to explain and publication of the measured energy spectrum up to $\mathrm{MeV}$ energies by DAMA is necessary to clarify this point. If a signal is present, then the background (measured spectrum minus signal) should have a minimum at about 1.5-2 keV. Its position and depth depend on the model but it is very hard to obtain such a minimum with simulations. Significantly enhanced concentrations of certain isotopes in the crystals help to bring the predicted rate and shape of the spectrum to agreement with the measured spectrum at low energies but leave too little room for the dark matter signal. Any dark mater model that predicts a large ratio (above 10 at $2-4 \mathrm{keV}$ ) of non-modulated (average) to modulated parts of the spectrum can certainly be excluded. The assumption of higher concentrations of certain isotopes, although improving agreement with the low-energy part of the spectrum, results in some features that are not seen by DAMA at high energies. We do emphasise that any interpretation of the annual modulation of the event rate observed by DAMA, as dark matter signal, is incomplete without clear understanding of the background spectrum.

Such integrated analysis would significantly restrict the dark matter models capable of explaining the modulation effect.

\section{Acknowledgments}

This work has been supported by the ILIAS integrating activity (Contract No. RII3CT-2004-506222) as part of the EU FP6 programme in Astroparticle Physics. We acknowledge the financial support from the Science and Technology Facility Council 
- STFC). We thank Prof. G. Gerbier and Dr. R. Lemrani (CEA-Saclay, France) for fruitful discussions and Dr. L. Pandola (LNGS-INFN) for helping us with generating radioactive decays with GEANT4. We are grateful to anonymous referees for useful comments.

\section{References}

[1] R. Bernabei et al. Phys. Lett. B, 424 (1998) 195.

[2] R. Bernabei et al. Phys. Lett. B, 480 (2000) 23.

[3] R. Bernabei et al. Eur. Phys. J. C, 56 (2008) 333; arXiv:0804.2741v1 [astro-ph].

[4] R. Bernabei et al. Nucl. Instrum. \& Meth. in Phys. Res. A, 592 (2008) 297; arXiv:0804.2738v1 [astro-ph].

[5] K. A. Drukier et al. Phys. Rev. D, 33 (1986) 3495.

[6] K. Freese et al. Phys. Rev. D, 37 (1988) 3388.

[7] M. Fairbairn and T. Schwetz, JCAP, 01 (2009) 037; arXiv:0808.0704v2 [hep-ph].

[8] C. Savage et al., JCAP, 04 (2009) 010; arXiv:0808.3607v3 [astro-ph].

[9] S. Chang, A. Pierce and N. Weiner, arXiv:0808.196v1 [hep-ph].

[10] G. J. Alner et al. (The UK Dark Matter Collaboration). Phys. Lett. B, 616 (2005) 17.

[11] M. J. Carson et al. Astroparticle Phys., 21 (2004) 667.

[12] M. J. Carson et al. Nucl. Instrum. \& Meth. in Phys. Res. A, 546 (2003) 509.

[13] V. Tomasello, V. A. Kudryavtsev and M. Robinson. Nucl. Instrum. \& Meth. in Phys. Res. A, 595 (2003) 431.

[14] M. Drees and G. Gerbier. Dark Matter, p. 235 in: W.-M. Yao et al., Review of Particle Physics, J. Phys. G, 33 (2006) 1.

[15] S. Agostinelli et al. (GEANT4 Collaboration), Nucl. Instrum. ES Meth. in Phys. Res. A, 506 (2003) 250.

[16] D. Budjas and L. Pandola. GERDA Report GSTR-07-010 (2007); L. Pandola, private communication.

[17] G. Gerbier et al. Astroparticle Phys., 11 (1999) 287.

[18] G. Gerbier. Talk at the 16th International Conference on Supersymmetry and the Unification of Fundamental Interactions - SUSY08 (June 16 - 21, 2008, Seoul, Korea); http://susy08.kias.re.kr/slide/pl/gerbier.pdf. 


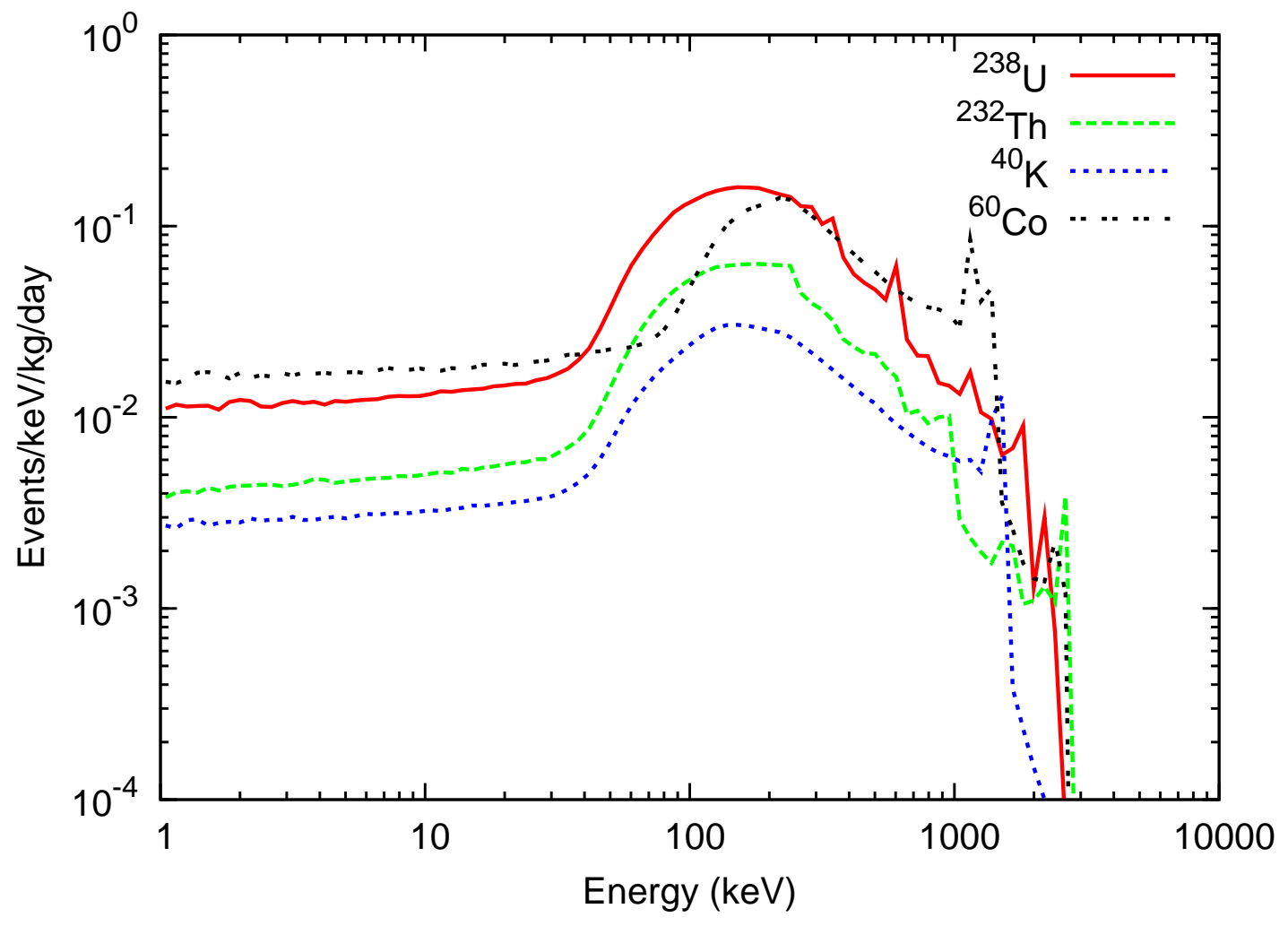

Figure 1: Spectra of energy depositions from electron recoils in the NaI crystals from ${ }^{238} \mathrm{U},{ }^{232} \mathrm{Th}$ and ${ }^{40} \mathrm{~K}$ decay chains in secular equilibrium. The source of radiation was the PMT envelopes (100 g each) attached to the light guides connected to the crystals. Also shown is the spectrum of ${ }^{60} \mathrm{Co}$ events from $\mathrm{Cu}$. Only events in which a single crystal was hit, are included. See text for details. 


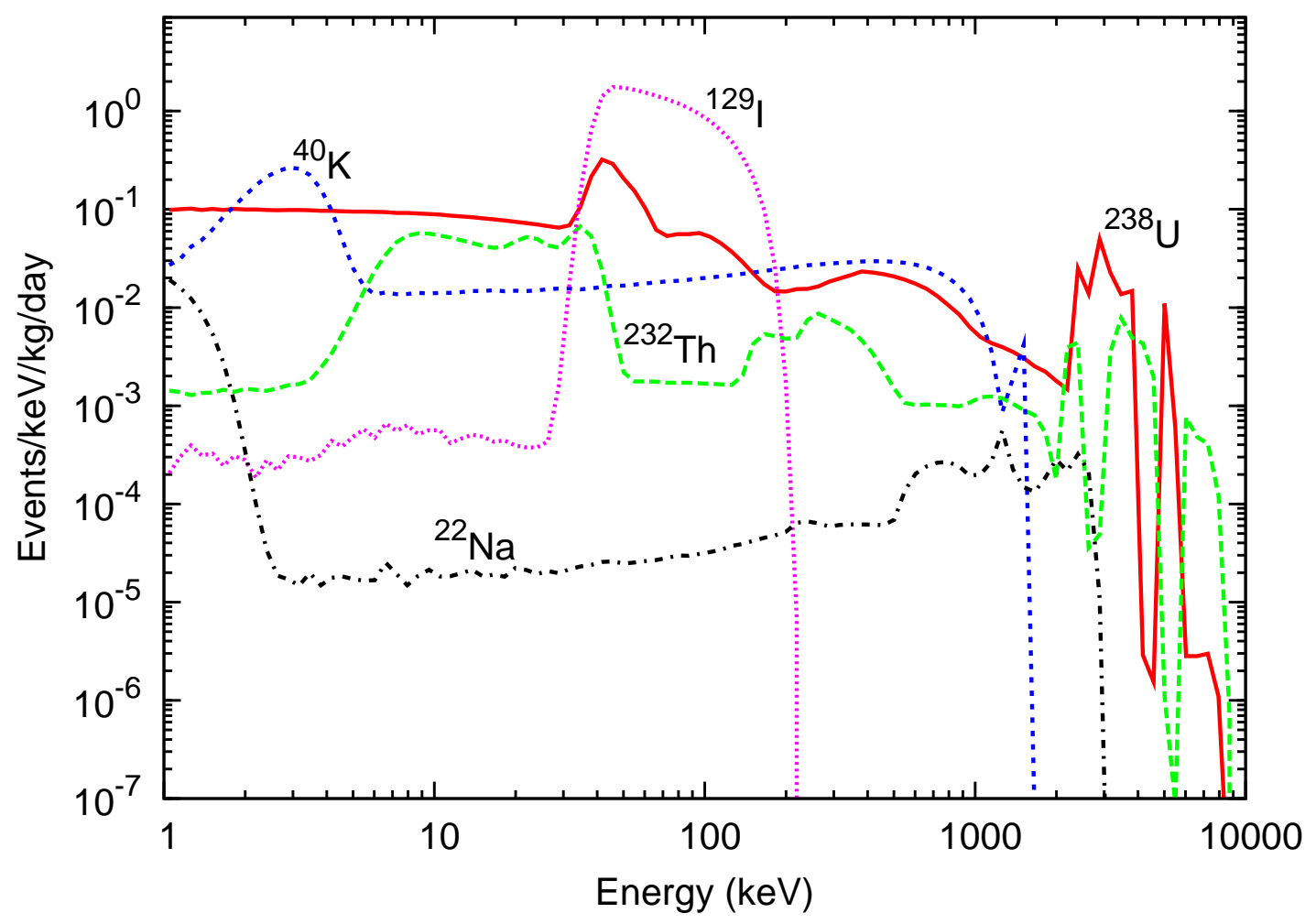

Figure 2: Spectra of energy depositions in the NaI crystals from ${ }^{238} \mathrm{U}$ (solid curve), ${ }^{232} \mathrm{Th}$ (dashed curve) and ${ }^{40} \mathrm{~K}$ (dotted curve) decay chains in secular equilibrium. The decays of ${ }^{129} \mathrm{I}$ and ${ }^{22} \mathrm{Na}$ were also included. Only events in which a single crystal was hit, are included. The decays of isotopes occurred within the sensitive volume of the crystals. Spectra were normalised using the typical concentrations reported in Ref. [4] 


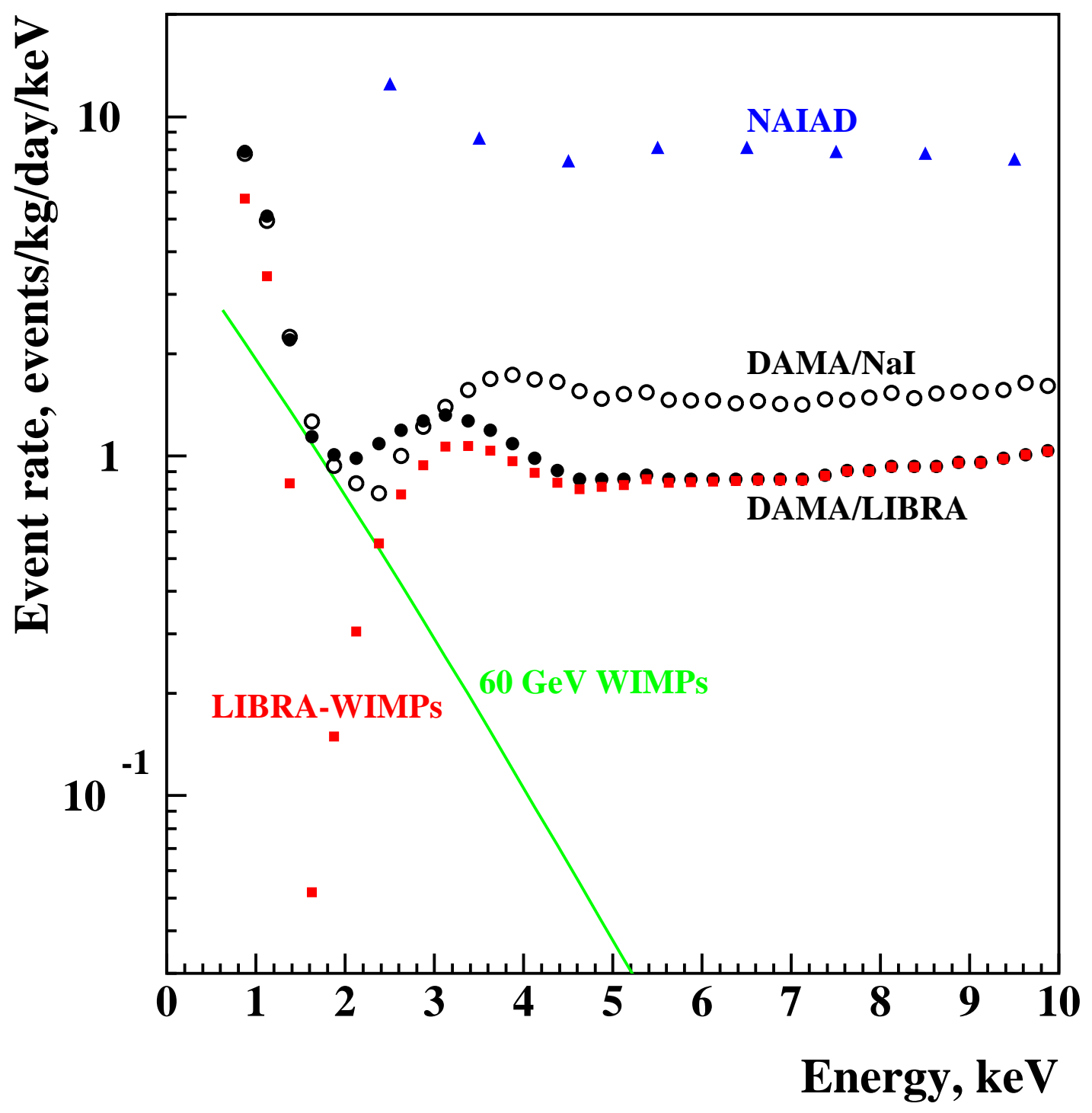

Figure 3: Energy spectra of single hit events as reported by the DAMA/NaI 2] (open circles) and DAMA/LIBRA [3] (filled circles) experiments. The spectrum of events expected from $60 \mathrm{GeV}$ WIMP interactions with the spin-independent cross-section of $7 \times 10^{-6} \mathrm{pb}$ in the isothermal halo model is shown as example by the solid curve (labeled as ' $60 \mathrm{GeV}$ WIMPs'). The difference between the measured DAMA/LIBRA spectrum and the WIMP signal is plotted as filled squares (labeled as 'LIBRA-WIMPs'). An example spectrum from one of the NAIAD crystals is shown by filled triangles. 


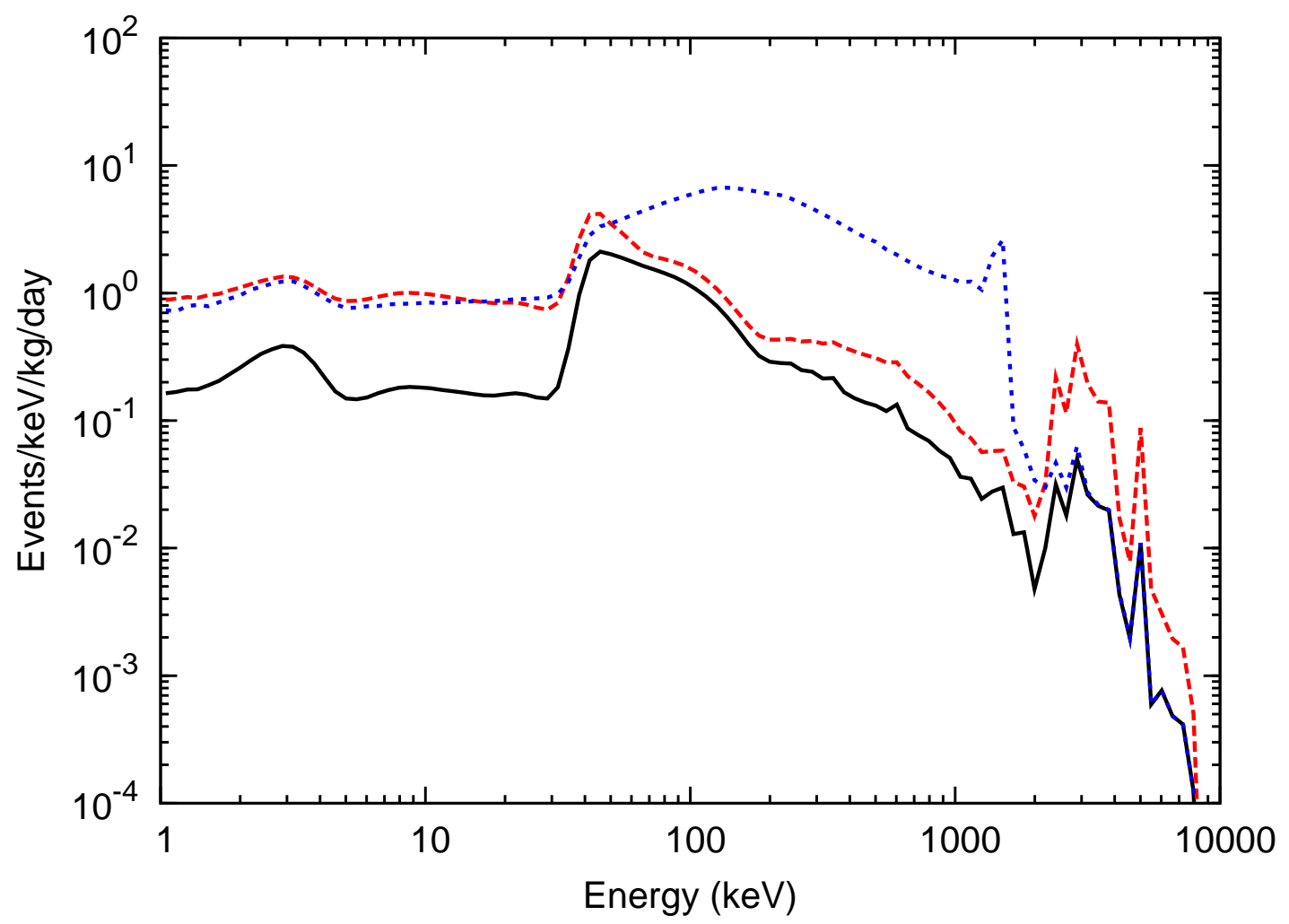

Figure 4: Combined spectra of energy depositions in the NaI crystals from radioactive background originating in the crystals and in the PMTs. Solid curve: PMTs $-30 \mathrm{ppb}$ of ${ }^{238} \mathrm{U}, 30 \mathrm{ppb}$ of ${ }^{232} \mathrm{Th}, 60 \mathrm{ppm}$ of natural potassium in $100 \mathrm{~g}$ of the PMT envelope; $\mathrm{NaI}-5$ ppt of ${ }^{238} \mathrm{U}, 5$ ppt of ${ }^{232} \mathrm{Th}, 10 \mathrm{ppb}$ of natural potassium, $0.2 \mathrm{ppt}$ of ${ }^{129} \mathrm{I}$ and $6.46 \times 10^{-14} \mathrm{ppb}$ of ${ }^{22} \mathrm{Na}$. Dotted curve: PMTs - U/Th - the same as for solid curve, $1.2 \%$ of $\mathrm{K} ; \mathrm{NaI}-\mathrm{U} / \mathrm{Th}$ - the same as for solid curve, $20 \mathrm{ppt}$ of K. Dashed curve: PMTs - the same as for solid curve; NaI - 20 ppb of natural potassium, 40 ppt of ${ }^{238} \mathrm{U}, 20$ ppt of ${ }^{232} \mathrm{Th}$, other isotopes - the same as for solid curve. 


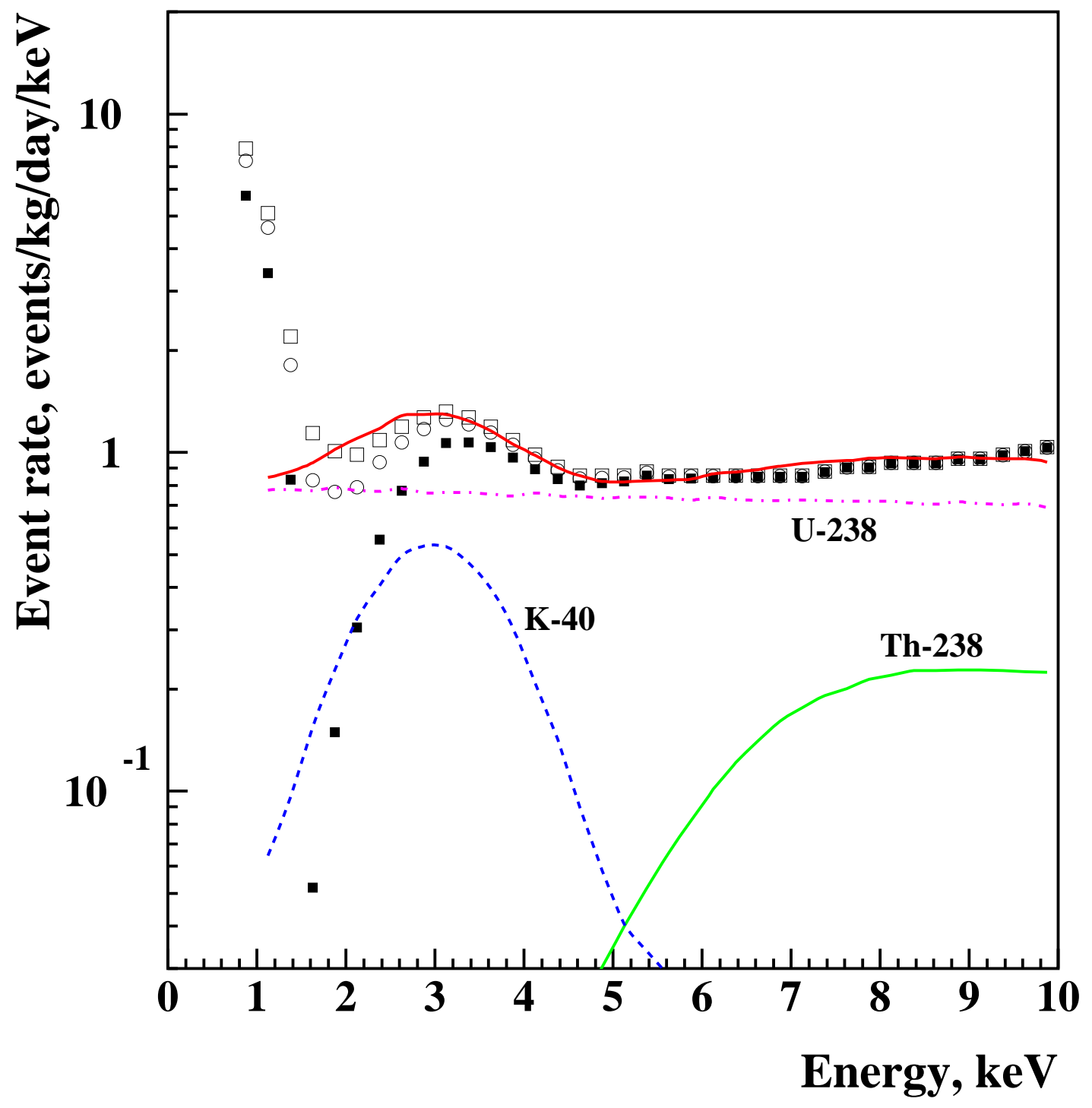

Figure 5: Simulated spectra of energy depositions in the NaI crystals from internal sources at low energies assuming 'optimised' concentrations of isotopes in the crystals (solid curve): $40 \mathrm{ppt}$ of ${ }^{238} \mathrm{U}$ (pink dashed-dotted curve), $20 \mathrm{ppt}$ of ${ }^{232} \mathrm{Th}$ (green solid curve), $20 \mathrm{ppb}$ of natural potassium (blue dashed curve), $0.2 \mathrm{ppt}$ of ${ }^{129} \mathrm{I}$ (not seen on the graph). The measured spectrum of DAMA/LIBRA without signal subtraction (open squares) and with signal subtraction are also shown. For the latter case we assumed a signal from $60 \mathrm{GeV}$ mass WIMPs with $\sigma_{S I}=7 \times 10^{-6} \mathrm{pb}$ (filled squares) and $\sigma_{S I}=2 \times 10^{-6} \mathrm{pb}$ (open circles) (see text for details). 


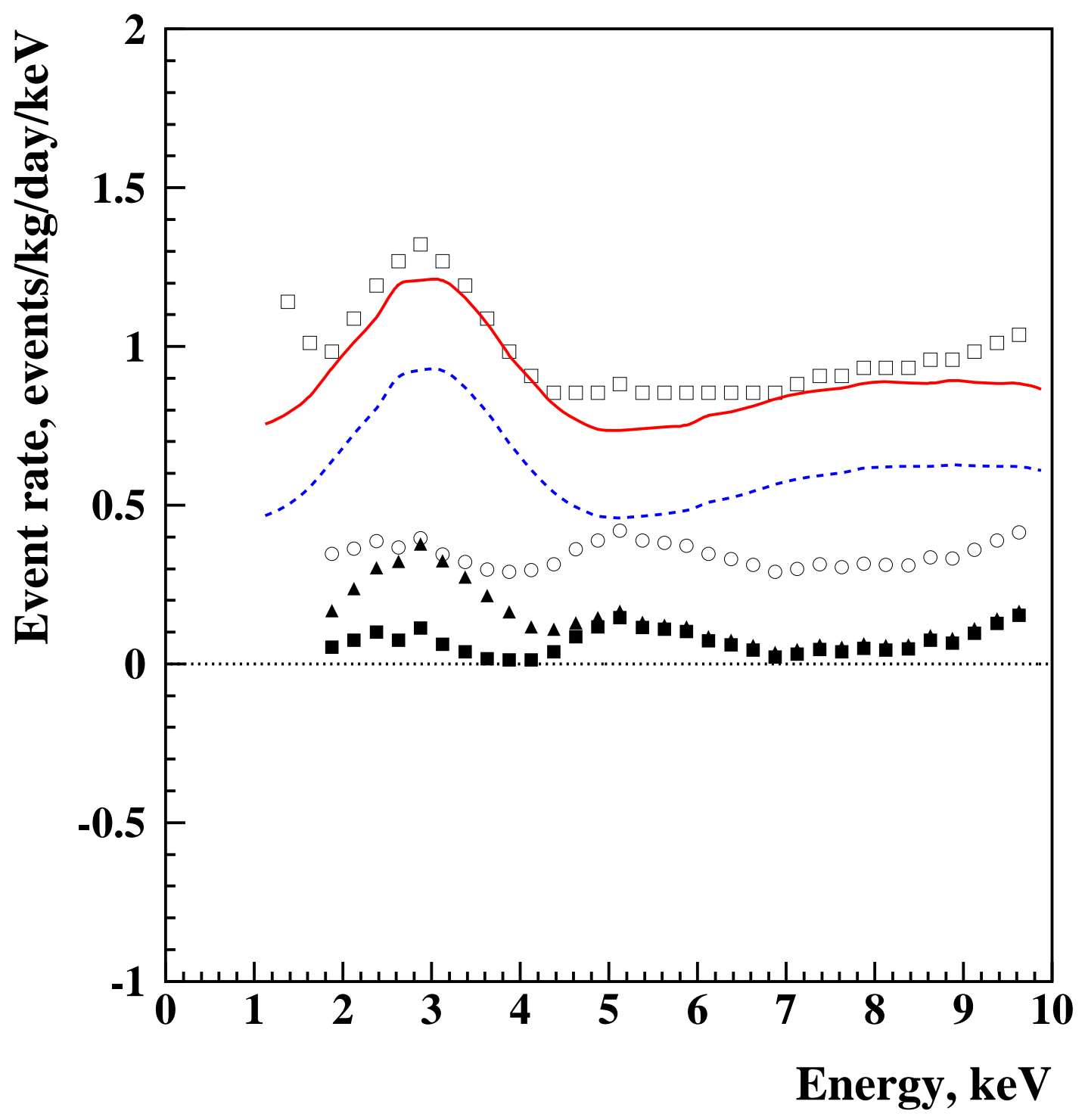

Figure 6: Simulated spectra of energy depositions in the NaI crystals from internal sources at low energies assuming 'optimised' concentrations of isotopes in the crystals (solid curve): $34 \mathrm{ppt}$ of ${ }^{238} \mathrm{U}, 20 \mathrm{ppt}$ of ${ }^{232} \mathrm{Th}$ and $20 \mathrm{ppb}$ of natural potassium. The measured spectrum of DAMA/LIBRA (open squares) is shifted by $0.25 \mathrm{keV}$ towards low energies to achieve better agreement with simulations around the $3 \mathrm{keV}$ peak. The difference between the two above spectra is shown by filled squares. The dashed blue line is the calculated spectrum assuming $20 \mathrm{ppt}$ of ${ }^{238} \mathrm{U}, 20 \mathrm{ppt}$ of ${ }^{232} \mathrm{Th}$ and $20 \mathrm{ppb}$ of natural potassium with the corresponding difference between the measurements and calculations shown by open circles. The difference between the measured spectrum and the simulated one assuming $34 \mathrm{ppt}$ of ${ }^{238} \mathrm{U}, 20 \mathrm{ppt}$ of ${ }^{232} \mathrm{Th}$ and $10 \mathrm{ppb}$ of natural potassium is denoted by filled triangles (see text for details). 